\title{
A JORDAN CURVE OF POSITIVE AREA*
}

BY

\section{WILLIAM F. OSGOOD}

The most general continuous plane curve without multiple points may be defined as a set of points which can be referred in a one-to-one manner and continuously to the points of a segment of a right line, inclusive of the extremities of the segment, if the curve is not closed; and to the points of the circumference of a circle, if the curve is closed. $\dagger$ Such a curve is called a Jordan curve. It may be represented analytically by the equations

$$
x=\phi(t), \quad y=\psi(t),
$$

where $\phi, \psi$ are single valued continuous functions of $t$ in the interval $0 \leqq t \leqq 1$ and where the equations

$$
x^{\prime}=\phi(t), \quad y^{\prime}=\psi(t)
$$

$x^{\prime}, y^{\prime}$ being given, admit at most one solution in the interval $0 \leqq t \leqq 1$, when the curve is not closed. In the case of a closed curve

$$
\phi(0)=\phi(1), \quad \psi(0)=\psi(1) ;
$$

otherwise, the above equations admit at most one solution.

JORDAN has shown that the area of a rectifiable curve is always null. $\neq$ But the question as to whether the exterior area $\S$ of a general Jordan curve is necessarily null has, so far as I know, never been answered.\| The example of this

* Presented to the Society December 30, 1902. Received for publication December 6, 1902.

† This is essentially Jordan's definition, Cours d'analyse, vol. 1, 2d ed., p. 90. The geometric formulation of the text is due to HuRwITz, Zürich Address, Verhandlungen des ersten internationalen Mathematiker-Kongresses in Zürich vom 9. bis 11. August 1897, Leipzig, 1898, p. 102.

$\ddagger$ loc. cit., p. 107, $\$ 112$. The area here meant is that of the curve regarded as a set of points ; not the area bounded by a closed curve, $c f$. inf.

8 The exterior area (cl. Peano, Applicazoni geometriche del calcolo infinitesimale, 1887, p. 156) may be defined as follows : Divide the plane into a network of squares whose sides are of length $2^{-n}$ and let $s_{n}$ be the sum of the areas of all those squares which contain in their interior or on their boundary a point of the curve. Then $s_{n}$ is a positive variable that decreases or remains constant as $n$ increases. Hence it approaches a limit when $n=\infty$. This limit, $I=$ limit $8_{n}$, is the exterior area in question.

|| JoRDAN raised a somewhat indefinite question as to whether the area of a curve $x=\phi(t), y=\psi(t)$ may be "indeterminate," and Peano answered by referring him to Peano's space-filling curve, Mathematische Annalen vol. 36 (1890), p. 157 ; cf. L'Intermediaire 
paper suffices to answer it in the negative. I was led to the construction of the curve through the consideration of some of the possibilities for the boundary of a simply connected region whose boundary points can all be approached along curves lying wholly in the region.*

We proceed to the consideration of the curve. It shall connect the extremities of the diagonal of a square and lie in the square. Begin with a square $S_{1}$, mark the extremities $A, B$ of a diagonal, and assume a system of Cartesian coördinates. $A$ and $B$ shall be respectively the first and last points of the curve, and the values of the parameter $t$ corresponding to them shall be $t=0, t=1$. Let the diagonal $A B$ be produced beyond the square in both directions. It will be convenient to consider the two regions $C$ and $D$ into which this line divides the part of the plane exterior to the square, and to shade these regions respectively blue and yellow. We may think of them as water and land.

First Step in the Construction.-Let $S_{1}$ be divided by canals and dykes of uniform width, as shown in Fig. 1 , into 9 equal squares. The canals consist of the blue shaded cross leading off from the region $C$, and a second short straight canal; a similar pair of dykes leads off from the yellow shaded region $D$ (the reflection of the canals in the diagonal $A B$ of the square), and is shaded yellow. The 8 lines in which a canal and a dyke come together form a part of the Jordan curve which we are constructing, and are marked in red. We will assign to each of them parametric values $t$ as follows.

Let the whole range of parametric values $0 \leqq t \leqq 1$ be divided into 17 equal intervals. Let a point $P$, starting from $A$, describe the whole boundary of the blue region pertaining to $S_{1}$. $P$ will first traverse two sides of the square numbered 1 ; for this square we will set aside the parametric values $0<t<1 / 17$ and dispose of them later. As $P$ proceeds, it next traverses a red line; to this line we assign the parametric values $1 / 17 \leqq t \leqq 2 / 17$, representing the coördinates of its points most conveniently as integral linear func. tions of $t, t$ increasing as $P$ advances.

Next, $P$ traverses two sides of the square numbered 2. For this square we will set aside the parametric values $2 / 17<t<3 / 17$ and dispose of them later. As $P$ proceeds, it now traverses a red line; to this line we assign the

des mathématiciens, vol. 1 (1894), p. 23, question 60 and vol. 3 (1896), p. 39 . But Prano's curve has multiple points, and hence is not a "Jordan curve." JüraENs has shown that a plane region cannot be mapped in a one-to-one manner and continuously on a segment of a right line; Jabresbericht der deutschen Mathematiker-Vereinigung, vol. 7 (1899), p. 50. As regards Peano's space-filling curve of. further Hilbert, Ueber die stetige Abbildung einer Linie auf ein Flächenstück, Math ematische Annal en, vol. 38 (1891), p. 459, and Mooke, On certain crinkly curves, Transactions Amer. Math. Soc., vol. 1 (1900), p. 72.

* For simply connected regions of which the above is not true, cf. OsGood, Transactions A mer. Math. Soc., vol. 1 (1900), p. 310. 


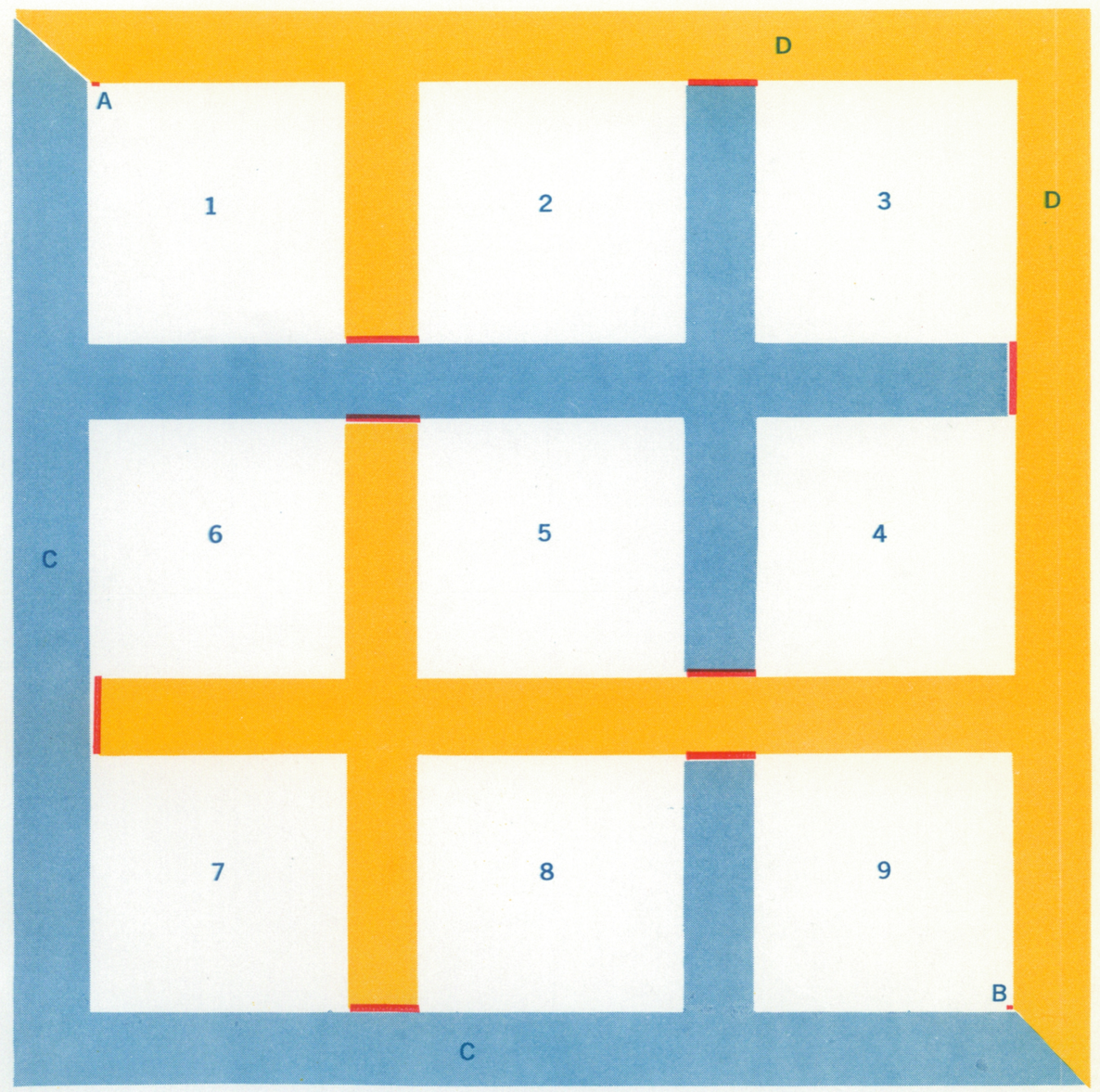

FIG. 1. 


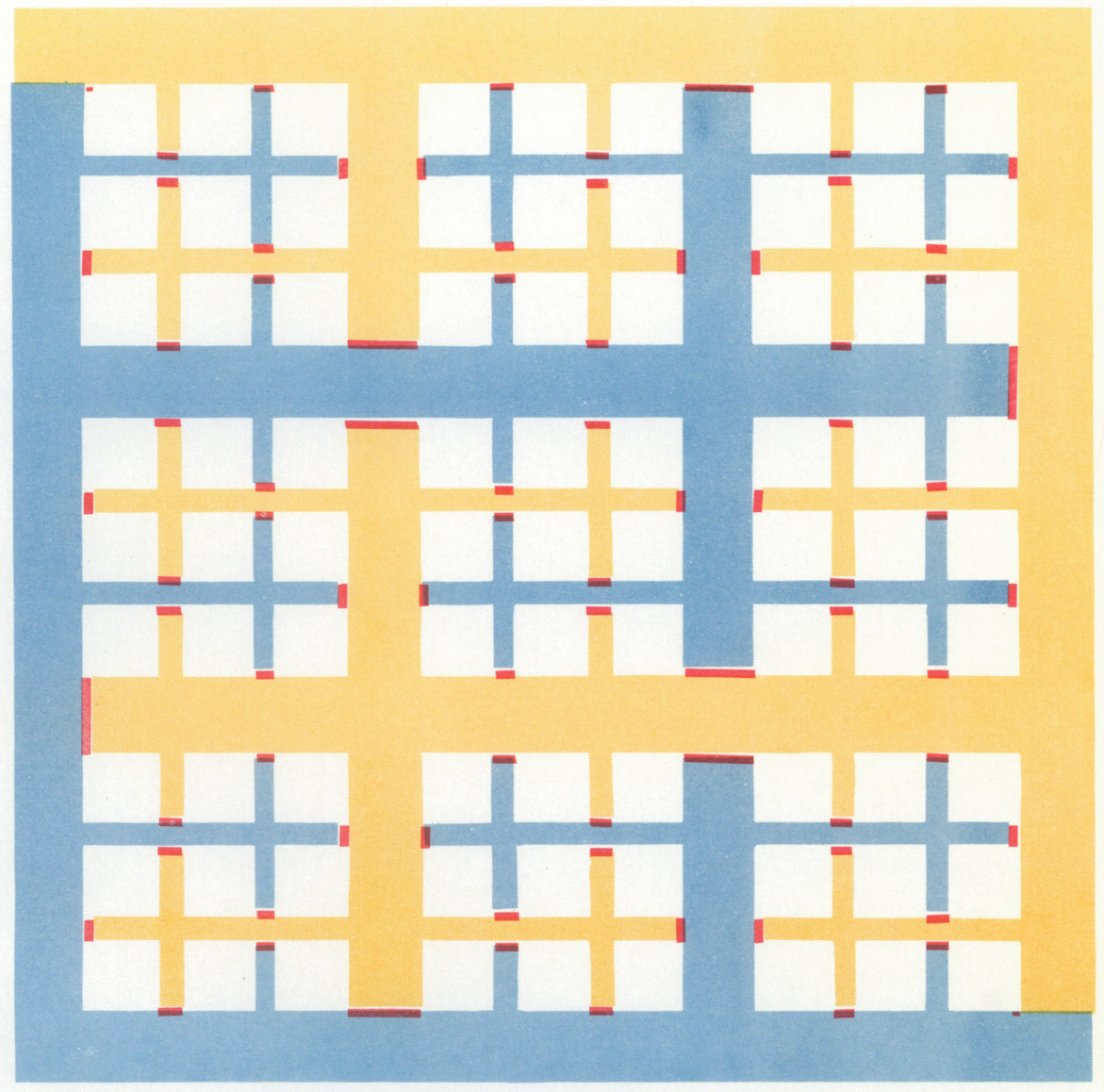

FIG. 2. 
parametric values $3 / 17 \leqq t \leqq 4 / 17$, representing the coördinates of its points most conveniently as integral linear functions of $t, t$ increasing as $P$ advances.

Repeating these steps, we finally arrive at $B$. Each one of the 8 pieces of the Jordan curve we are constructing has been represented in parametric form :

$$
\begin{gathered}
x=\phi(t), \quad y=\psi(t), \\
\frac{2 n-1}{17} \leqq t \leqq \frac{2 n}{17}
\end{gathered}
$$$$
(n=1,2 ; \cdots 8) \text {. }
$$

Hitherto we have said nothing about the width of the canals and dykes, except that it is to be uniform. We will take the area of the square $S_{1}$ as unity and determine the breadth of the canals and dykes so that their total area shall have the arbitrary value $\alpha_{1}<1$.

Second Step in the Construction.-The second step consists in constructing canals and dykes in each of the small squares, similar to those just constructed in $S_{1}$, and in taking the lines along which a canal and a dyke come together as further lines of the Jordan curve. These lines will be marked in red. In detail the process is as follows: We observe first of all that each one of the squares numbered $1 \ldots 9$ is bounded on two adjacent sides by canals and on the other two sides by dykes. If, then, the diagram of the canals and dykes of $S_{1}$ is suitably reduced in scale and, if necessary, turned over, it may be placed in a given one of the nine squares, and serve as a plan for laying out new canals and dykes. We consider this as done in each of the nine squares, cf. Fig. 2.* Let us fix our attention on the square numbered 1. We had set aside for this square the range of parametric values $0<t<1 / 17$. This range we divide into 17 equal parts. As in the case of $S_{1}$; so here, we have nine smaller squares and eight segments of right lines (the red lines). We allow a point, starting from $A$, to describe the whole boundary of the blue region pertaining to this square. We represent the 8 red lines in parametric form as follows :

$$
\begin{gathered}
x=\phi(t), \quad y=\psi(t), \\
\frac{2 n-1}{17^{2}} \leqq t \leqq \frac{2 n}{17^{2}}
\end{gathered}
$$$$
(n=1,2, \cdots 8)
$$

and we set aside for each of the smaller squares respectively the complementary parametric intervals. - Similarly for each of the squares numbered 2, 3..9. The total blue shaded region is connected-in fact is a simply connected region; and likewise the yellow shaded region. This completes the second step in the construction of the curve, except for one point; the breadth of the canals is arbitrary, and shall be determined as follows.

\footnotetext{
* Owing to an oversight the boundary between the blue shaded and the yellow shaded regions outside the square $S_{1}$ does not follow the diagonal of the square, produced, as in Fig. 1 . It should follow this diagonal, as in Fig. 1.
} 
Let a positive number $\lambda<\frac{1}{2}$ be chosen at pleasure, and let

$$
\epsilon_{1}+\epsilon_{2}+\cdots \quad\left(\epsilon_{n}>\epsilon_{n+1}\right),
$$

be a convergent series of positive terms whose value is $\lambda$. Then $I$ will choose the breadth of the canals and dykes laid out in $S_{1}$ at the first step of the construction so that the total area of the canals shall be

$$
\frac{1}{2} \alpha_{1}=\epsilon_{1} \text {. }
$$

In each of the squares $1 \ldots 9$ the canals and dykes laid out in the second step of the process shall have such a breadth that the area of the canals in a given square will be $\frac{1}{9} \epsilon_{2}$, and hence the total area for all 9 squares will be $\epsilon_{2}$.

The n-th Step. - It is now clear how the process of construction of the curve is to proceed. We are to lay out, as the third step in the process, canals and dykes in each of the $9^{2}$ white squares and we are to choose their breadths so that the total area for all the new canals will be $\epsilon_{3}$. Thus we get $8 \times 9^{2}$ new segments of the curve, each segment being represented in parametric form, while for each one of the $9^{2}$ new white squares is set aside a range of parametric values, to be disposed of later.

Let the step be repeated indefinitely often. W.e thus get an enumerable set of (red) segments of right lines, each represented in parametric form. The corresponding ranges of parametric values form a set of segments $M$ lying in the interval $0 \leqq t \leqq 1$ and having certain points of condensation.*

The Remaining Points of the Curve. - The last step in the construction of the curve now suggests itself, namely, to adjoin to the curve all the points of condensation of the red segments not already belonging to the curve, assigning to each that parametric value which is the limit of the parametric values pertaining to those red segments which cluster about the point in question. That such a limit exists is clear from the fact that such a point $P$ is at each step in the construction of the red segments an interior point of a white square. Let a positive quantity $\eta$ be chosen at pleasure. Then it is possible to mark off a neighborhood of $P$-namely the interior of the white square in which $P$ lies when the $m$ th step in the construction of the red segments has been takensuch that the parametric values $t$ assigned to the red segments of this neighborhood all lie in an interval

$$
t_{m}^{\cdot}<t<T_{m}, \quad T_{m}-t_{m}<\eta .
$$

For, we need only to choose this interval as the range of parametric values set aside for this square. Thus it appears that to the point $P$ corresponds one value $t$ which is the limit approached by the values of $t$ pertaining to red seg-

* It is readily seen that the content of the set of points of condensation of the extremities of their segments is null. 
ments which cluster about $P$. This value of $t$ we assign then to $P$, and thus to each value of $t$ in the interval $0 \leqq t \leqq 1$ corresponds a single point of $S_{1}$, two different values of $t$ always leading to distinct points of $S_{1}$.

It remains to show that the functions

$$
x=\phi(t), \quad y=\psi(t), \quad 0 \leqq t \leqq 1,
$$

thus defined are continuous for all values of $t$ in the interval $(0,1)$. If $t$ is an interior point of one of the parametric ranges $M$, then the corresponding point $(x, y)$ is an interior point of a red segment, and $\phi, \psi$ are continuous by their definition. If $t$ is not such a point, then a neighborhood of the corresponding point $(x, y)$ may be marked off such that the parametric values of $t$ corresponding to the points of the curve lying in it will differ arbitrarily little from the value of $t$ in question, and will completely fill a certain neighborhood of this value. That two distinct values of $t$ lead to two distinct points of the curve is evident.

The Jordan curve is now complete. Its exterior area $A$ is $1-2 \lambda$. For, as is readily shown, the interior area $B$ of the canals is $\lambda$; similarly, the interior area $\mathbf{C}$ of the dykes is $\lambda$; and

the area of $S_{1}$.

$$
A+B+C=1,
$$

In the neighborhood of every point of the curve not an interior point of a red segment there lie an infinite number of points of the curve about which the curve coils like a logarithmic spiral an infinite number of times. It would not be difficult to modify the construction in such a manner that the red segments shrink down to points and that the new curve has an infinite number of such spiral vertices in the neighborhood of every one of its points. Such a curve would not have a tangent in any one of its points. It may furthermore be remarked that Peano's space-filling curve above cited may be obtained as the limit of our curve when the breadth of all canals and dykes is allowed to approach 0 as its limit. The assignment of parametric values must then be slightly modified.

An Analogous Closed Jordan Curve. - As I said at the beginning of the paper, I was led to the construction of this curve by considering some of the possibilities for the boundary of a simply connected region. I did not think of this region as the blue shaded region $C$ of the plane and the canals above employed, but I started with a region lying in a circle, its boundary being inclosed in a circular ring. Let such a ring be divided by radii into an even number of equal quadrilaterals, the radii of the ring being so chosen that such a quadrilateral may be mapped conformally on a square, the square $S_{1}$, vertices corresponding to vertices. Then the Jordan curve that we have constructed 
goes over into a curve coursing the corresponding quadrilateral of the circular ring. Let the latter curve be reflected in a radius through one of its extremities, and by a series of such reflections constructed in each of the quadrilaterals. The result is a closed Jordan curve of positive external area, bounding completely a simply connected region contained within the outer circle. The closed Jordan curve separates the points of the plane into interior and exterior points, but the interior area of the interior of the curve is not the same as its exterior area. It was through the construction of such a simply connected region that I was led to the Jordan curve above described.

HARVARD UNIVERSITY,

Thanksgiving, 1902. 